\title{
Quality of Life Protocol in the Early Asthma Diagnosis in Children
}

\begin{tabular}{|r|l|}
\hline Journal: & Pediatric Pulmonology \\
\hline Manuscript ID: & PPUL-09-0111.R4 \\
\hline Wiley - Manuscript type: & Original Article \\
\hline Autho Submitted by the & 05-May-2010 \\
\hline Complete List of Authors: & $\begin{array}{l}\text { Farnik, Małgorzata; Silesian University of Medicine, Pneumonology } \\
\text { Pierzchała, Władysław; Silesian University of Medicine, } \\
\text { Pneumonology } \\
\text { Brożek, Grzegorz; Silesian University of Medicine, Epidemiology } \\
\text { Zejda, Jan; Silesian University of Medicine, Epidemiology } \\
\text { Skrzypek, Michał; Medical University of Silesia, Department of } \\
\text { Biostatistics }\end{array}$ \\
\hline Keywords: & asthma, quality of life, early diagnosis, child \\
\hline
\end{tabular}

\section{s scholaroNE" \\ Manuscript Central}




\section{Quality of Life Protocol in the Early Asthma Diagnosis in Children}

Małgorzata Farnik MD PhD1, Władysław Pierzchała MD PhD, Grzegorz Brożek MD $\mathrm{PhD}^{2}$, Jan E. Zejda MD PhD², Michał Skrzypek $\mathrm{MS}^{3}$.

1 Department of Pneumonology, Medical University of Silesia, Katowice, Poland

2 Department of Epidemiology, Medical University of Silesia, Katowice, Poland

${ }^{3}$ Department of Biostatistics, Faculty of Public Health, Medical University of Silesia, Bytom, Poland

Correspondence: Małgorzata Farnik MD PhD

Department of Pneumonology

Medical University of Silesia

Ul. Medyków 14

40-752 Katowice, Poland

tel/fax. 48-32-252-38-31

e-mail pneumo@sum.edu.pl

Key words: asthma, pediatrics, health-related quality of life, early diagnosis

Abbreviated title: QOL in early asthma diagnosis 


\section{Summary}

Introduction. Asthma is considered an important problem in children and influences on their everyday functioning. This study was concerned with the impact of asthma on the health related quality of life (HRQOL) of newly recognized pediatric asthma patients and their parents.

Methods. Patients were identified by a general respiratory survey. HRQOL was assessed by self-report measures: disease-specific questionnaire for children, the Pediatric Asthma Quality of Life Questionnaire (PAQLQ) and generic for parents - the Quality of Life in Child's Chronic Disease Questionnaire (QLCCDQ).

Results. 29 newly diagnosed asthmatic children aged 7-11 years (mean age $=8.6$ $S D=1.4$ ) were identified from a population-based respiratory health survey. The most impaired HRQOL domains differed in parents and children. In children, activity limitation was the most impaired; however in parents, emotional functioning and symptom perception domains were most impaired. There were no statistically significant differences in patient and parental HRQOL observed in either the group where symptoms were present or in the group were symptoms were not present. Baseline spirometry showed correlation for $\mathrm{FEV}_{1} \% \mathrm{FVC}$ and PAQLQ symptom domain $(r=0.42)$. Exercise test showed correlations between $F E V_{1} \% F V C$ and QLCCDQ emotional domain ( $r=0.7)$; MEF25-75\% and family roles $(r=0.81)$, as well as social functioning $(r=0.81)$. Activity limitation on the PAQLQ correlated with the MEF $25-75 \%(r=0.44)$ and PEF $(r=0.55)$.

Conclusions. HRQOL newly recognized children with asthma and their parents does not correlate closely with clinical status. Patient and parental HRQOL should be considered as independent dimensions. 


\section{Introduction}

The results of International Study of Asthma and Allergies in Childhood (ISAAC) have shown that childhood asthma represents an important public health problem worldwide (1). Previously increasing prevalence of childhood asthma, well documented in many countries $(2,3,4)$ can be observed in Eastern Europe over the years $1994-2007(1,5,6,7)$. The magnitude of the problem could be underestimated because of underdiagnosis (8). The resulting public health burden justifies the development and implementation of population-based measures. Asthma not only has a profound impact on public health, but also on the everyday lives of individual patients.

A patients' burden of chronic illness cannot be assessed by disease severity; it requires measurement reflecting personal perception. There is an increasing interest in activity limitation, emotional functioning, impact on every-day life due to disease and the implementation of health-related quality of life (HRQOL) questionnaires. Deterioration of HRQOL has been described in children and adults with allergic diseases, such as eczema (9) and rhinitis (10). HRQOL assessment highlights important and troublesome problems experienced by patients with asthma $(11,12,13)$. Children with asthma suffer from activity limitation, emotional problems such frustration, fear and irritation (14). Although HRQOL does not correlate closely with asthma control, it is known that children with very severe asthma tend to have a poorer quality of life than those suffering from mild or moderate condition (15). Parents of children with asthma could be limited in normal daily activities and experience emotional distress (16). 
Clinical experience shows that a caregiver's HRQOL and their perception of a child's symptoms are important in diagnosis and control of established asthma (17). Less is known about the potential impact of disease on HRQOL in newly recognized children with asthma which were found as the result of screening programs. It remains unknown to what extent such a procedure could be helpful in both early assessment of asthma and its impact on patients' and parental quality of life.

We implemented the HRQOL protocol in examination of newly identified pediatric asthma patients and their families, participants of a large population-based respiratory health survey. The goal of the study was to determine the impact of asthma on the health related quality of life of newly recognized pediatric asthma patients and their parents. 


\title{
Materials and Methods
}

\begin{abstract}
A large population-based respiratory health survey resulted in identification of new pediatric asthma patients. All newly identified children with asthma aged 7-11 years and their parents were involved in HRQOL study. A disease-specific quality-oflife questionnaire was used for the children and a generic questionnaire for parents. Clinical status data was obtained prior to an HRQOL assessment.
\end{abstract}

\section{$\underline{\text { Participants }}$}

Children were recruited using cluster sampling during a respiratory health survey study in primary schools in Katowice (Southern Poland). 2254 children grades I-IV, in 7 out of 51 schools in the town were invited $(17.0 \%$ of the town population in the defined age group) for respiratory questionnaire assessment. This was a validated questionnaire, used in The Central European Study of Air Pollution and Respiratory Health (CESAR) [Table 1] and other surveys $(22,23,24)$. The response rate was $80.8 \%$ (1822 children) . Study design is shown in Fig. 1 Figure 1. Study design

Based on the questionnaire results children were categorized into three mutually exclusive groups: "Asthma" (5.4\% - diagnosis of asthma established before the survey); "Respiratory Symptoms" (27.9\% - no previous diagnosis of asthma and one or more symptoms of persistent cough excluding infections, chest wheezing, dyspnea during the last 12 months), "No Respiratory Symptoms" (66.7\% - no previous diagnosis of asthma and no respiratory symptoms during the last 12 months). 
[Table 1] - Respiratory Questionnaire (examples of questions)

Clinical evaluation was proposed to all children in "Asthma"- group and 60\% samples from groups "Respiratory Symptoms" and "No Respiratory Symptoms", with the response rates: $41.4 \%, 70.8 \%$ and $25.6 \%$, respectively. A certified pulmonologist (Department of Pneumonology, Medical University of Silesia) examined 415 children. The physician was blind to the results of the questionnaire. Diagnostic procedures were based on GINA guidelines for childhood asthma (25). Forced expiratory volumes were measured according to ERS/ATS recommendations (26). Spirometry was considered normal if $\mathrm{FEV}_{1}, \mathrm{FVC}$ and PEF values exceeded $80 \%$ predicted and if $\mathrm{FEV}_{1} \% \mathrm{FVC}$ ratio was greater than $75 \%$. If actual values presented predicted values, a further challenge test of bronchial hyperresponsiveness, described by Gerald et al (27), was used. A sub-maximal exercise test (stepping up and down on a single step with metronome rhythm) was followed by spirometry obtained at minutes 3,5 and 10 after exercise. An $\mathrm{FEV}_{1}$ decrease greater than $15 \%$ was considered positive for asthma. Medical records were provided; additional skin allergy tests and a chest radiograph were carried out, where needed.

Clinical assessment of 415 children confirmed 33 previously known and revealed 29 new cases of asthma. All newly identified children with asthma and their parents were invited to HRQOL assessment.

\section{$\underline{\text { HRQOL measurements }}$}

Quality of life questionnaires were completed by children with asthma at school in the patient's normal psychosocial environment. Parents of newly diagnosed children 
with asthma completed HRQOL questionnaire as well at school, before the diagnosis was communicated.

The Paediatric Asthma Quality of Life Questionnaire

The Paediatric Asthma Quality of Life Questionnaire (PAQLQ), dedicated to children aged 7-17 years old, was used as a well-validated and reliable measure of HRQOL in childhood asthma. The questionnaire consists of 23 questions, covering symptoms, emotional function and limitations of activity. Children were asked to recall their experiences over the previous week and responded on a 7 - point scale (7 - no impairment, 1 - severe impairment). Patients completed the questionnaire with physicians experienced in HRQOL research. Interviewer-administration was used to avoid parental interference with patient responses.

\section{Quality of Life in a Child's Chronic Disease Questionnaire}

An accompanying questionnaire for parents was devised by the authors, based on standard procedure - starting from initial questions (identification of needs, definition of operational objectives), tool development, pretesting and final version (format, wording) preparation, pilot testing and validation of the questionnaire $(19,20)$. Quality of Life in a Child's Chronic Disease Questionnaire (QLCCDQ) is concerned with daily problems and limitations that concern parents of children with chronic disease. QLCCDQ is a self - report measure of parent's HRQOL, only symptoms subscale is parent-proxy measure. Most questions concern parents' functioning, only the symptom subscale reflects parents' perception of symptoms presence. QLCCDQ consisted of 15 questions and covered patient-perceived symptoms (e.g Did the child show any worrying symptoms?), emotions (e.g. Have you experienced anxiety because of your child's health problems?) and activity limitation in roles function: social (e.g. Please indicate how much you have been limited by your child's 
disease in listed activities in past two weeks: going to church, cinema, visiting friends), family roles (e.g. Do you struggle to find time to spend with other family members (spouse, another child) because of your child' disease?) and occupational (e.g. Please indicate how much you have been limited by your child's disease in work related activities in past two weeks).

The questionnaire required circling a choice from seven responses devised for each question, from 1 (most bothered or limited) to 7 (not bothered or limited). All questions concerned parental HRQOL of previous two-week period.

\section{Psychometric data}

\section{PAQLQ}

The instrument is reproducible $(\mathrm{ICC}=0.95)$ and has shown ability to detect HRQOL changes as a result of treatment $(p<0.001)$. Estimation of reliability in stable asthma has shown that it was consistent throughout all domains (ICC: 0.84-0.93).

\section{QLCCDQ}

Reproducibility based on the weighted kappa coefficients showed an expected level of agreement. Values of weighed kappa coefficients suggested almost perfect agreement $(0.81-1.0)$ in case of 8 questions, substantial $(0.61-0.8)$ for 5 questions and moderate $(0.41-0.6)$ for 2 questions. The reliability of the defined subscales was evaluated by means of Cronbach's $\alpha$. The values of $\alpha$ ranged between 0.74 and 0.94: symptoms: 0.93 , emotions: 0.91 , family roles: 0.77 , social roles: 0.79 , occupational roles: 0.92 and activity limitations in roles function: 0.91 .

\section{$\underline{\text { Child's school grades and absenteeism }}$}


Supplementary data such as school grades and absenteeism (missed school days due to asthma symptoms) were provided to determine if there were relationships between reported HRQOL and child's functioning at school. It could be expected that untreated asthma has an impact on school functioning resulting in children lower school grades and missed school days. Polish school grades assessment is based on 6- point scale: from 1 (Fail) to 6 (Excellent).

The study protocol was approved by the ethics committee (Medical University of Silesia). Each child admitted to the study had the written consent of a parent.

\section{Statistical analyses}

The statistical analysis was performed using standard procedures available in the Statistica 7.1 package and SAS, version 9.2 (SAS Institute Inc., Gary, NC). Normality of distributions of continuous variables was analyzed using the ShapiroWilk test. Statistical significance of differences between continuous variables was analyzed by t-Student test and ANOVA while for data with non-normal distribution, $U$ Mann-Whitney and Kruskal-Wallis tests were used. Differences between categorical variables were examined by the chi-square test. Strength of associations, due to nonnormal distributions, were measured by Spearman's rank correlation coefficient.

The statistical inferences were based on confidence levels $p<0.05$. If multiple comparisons were used statistical significance was lowered according to the Bonferroni adjustment. 
Variables included patient and parental HRQOL assessment, symptoms over the previous 12 months, baseline and exercise spirometry, school grades and absenteeism.

Correlations between child's and parental HRQOL scores were analyzed. Relations between clinical status (symptoms, lung function tests) and HRQOL were examined, as well as links between educational markers (school grades and absenteeism) and patient HRQOL scores. Gender and age effects on HRQOL were also analyzed. 


\section{Results}

\section{Participating children}

In children with newly diagnosed asthma (mean age $=8.6$ years; $S D=1.4$ years), classified according to GINA protocol, the disease was either episodic (20 children: 6 boys and 14 girls) or chronic mild ( 9 children: 4 boys and 5 girls). New diagnoses of asthma were made in $10.9 \%$ of children from the "Respiratory Symptoms" group and in $3.6 \%$ of children from the "No Respiratory Symptoms" group. All 29 newly diagnosed children with asthma and 21 parents responded to the subsequent HRQOL questionnaire.

[Table 2 ] - Demographic and asthma severity

\section{Patient and parental HRQOL}

HRQOL was assessed for each domain, the subscale mean ranging from 1.0 to 7.0. Lower HRQOL scores reflected greater impact of disease on every-day functioning. Children's most affected HRQOL domain was limitation of activity. Parents' impairment was most severe in emotional functioning and perceived symptoms, while less impaired domains included social functioning. Domains and overall HRQOL scores for children and parents appear in the table 3.

[Table 3]. HRQOL - domains and overall scores

Statistically important positive correlations were observed between all PAQLQ subscales, as well as most QLCCDQ subscales [ Table 4]. QLCCDQ symptom perception subscale correlated only with parental emotional functioning, while 
correlations were found between most subscales describing roles functioning.

Bonferroni correction suggested significance level was $p<0.0001$.

[Table 4]. PAQLQ and QLCCDLQ subscales Spearman's correlations

\section{$\underline{H R Q O L}$ measures and clinical assessment}

Symptoms

The differences of mean QLCCDQ and PAQLQ subscales scores between groups of symptomatic and nonsymptomatic children over in the past 12 months were analyzed. Both for QLCCDQ and PAQLQ the differences in scores did not reach statistical significance. Bonferroni correction suggested significance level was $p<$ 0.01 .

\section{Lung function tests}

Statistically important correlations were found between HRQOL and spirometry. Challenge tests were done in 23 of the 29 children -3 children did not obtain reproducible spirometry after exercise, 2 children were wheezing before exercise commenced, 1 child refused to cooperate. Baseline FEV ${ }_{1} \% \mathrm{FVC}$ and symptom domain in children showed a correlation $(r=0.42)$, while exercise value $\mathrm{FEV}_{1} \% \mathrm{FVC}$ correlated with emotional domain ( $\left.r=0.7\right)$. MEF25-75\% correlated with QLCCDQ family roles $(r=0.81)$ and social functioning $(r=0.81)$. PAQLQ activity limitation was correlated with MEF 25-75\% $(r=0.44)$ and PEF $(r=0.55)$, both obtained during the exercise test. All exercise test correlations were based on spirometry results collected 10 minutes after the challenge test [Table 5].

[ Table 5]. HRQOL scores and lung function - Spearmans' correlations 


\section{School grades and absenteeism}

Higher scores on the symptoms subscale of PAQLQ correlated with better school grades $(r=0.64 ; p=0.02)$ and fewer missed school days $(r=-0.53 ; p=0.02)$. No correlations were observed in parents' HRQOL and missed school days or school marks.

\section{Patient gender and age}

Females showed a significantly lower symptom score in comparison to males $(p=0.01)$, however this effect was not translated into total PAQLQ scores nor into parents' HRQOL.

The relationship between HRQOL scores and age were not statistically significant. 


\section{Discussion}

Our study focused on the implementation of the HRQOL protocol in early diagnosis of childhood asthma. The analysis involved data obtained in children with newly diagnosed asthma and in their parents. Since asthma cases were identified through population-based screening, it is not surprising that all previously undiagnosed cases represented either episodic or mild asthma - children with more advanced disease had been diagnosed on regular basis, via pediatric health care. An important finding of our study is that the profile of HRQOL impairment is different in children with asthma and in their parents. Not only did PAQLQ and QLCCDQ scores not correlate but - in addition - the highest impaired domains differed between children and parents. Symptom perception and emotional functioning appeared to be the most affected HRQOL domains in parents, while activity limitation was the most impaired domain in children. Some parents might limit their child's activity, due to emotional distress caused by anticipation of symptoms, and such behavior could explain the lack of correlation between emotion domain QLCCDQ and symptom or activity limitation domains in PAQLQ.

It is of interest that parental HRQOL pattern is in line with the published qualitative evidence that parents tend to perceive symptoms of asthma in a child as intermittent acute episodes rather than a chronic condition (28). Such interpretation could explain the lack of diagnosis of episodic or mild asthma in our study. It cannot be excluded that such a presentation is not entirely neglected by parents, but treated by them as an annoying condition and not overt disease. Our finding could have some implication as far as screening is concerned - occurrence of symptoms is necessary 
but does not have to be a sufficient cause to seek medical advice. Other important factors include individual experience, perception and emotions.

The relationships between clinical indices and HRQOL were analyzed in many studies as an important observation for clinicians $(29,30,31,32)$. Our study has shown that there were no statistically significant differences in both patient and parental HRQOL scores between symptomatic and asymptomatic groups. However some of analyzed relationships were suggested as significant; before the significance level was lowered by applying Bonferroni correction to $p<0.01$. As the example compared to parents of asymptomatic children, parents of children complaining of wheezing had a lower $(p=0.02)$ mean score in emotional function and symptom domain. As well the group of children possessing dyspnea showed a lower mean parental emotional function score $(p=0.04)$ than in asymptomatic children. In children an emotional function score was lower in the group of children complaining of cough in the past 12 months $(p=0.01)$ as compared to asymptomatic children.

Correlations were observed for exercise lung function and QLCCDQ domains, such as emotional, family and social functions. Worthy to note, that the correlations between children's lung function and parental HRQOL domains were stronger than analogous correlations concerning HRQOL in children. The most impaired parent HRQOL domain - emotions, significantly correlated with FEV $1 \%$ FVC. Activity limitation, the most impaired patient domain, correlated with PEF and MEF 25-75. Even if the correlations between clinical indices and HRQOL scores were observed only for few variables it should not determine a limitation of HRQOL protocol in early diagnosis. As HRQOL is not a diagnostic scale nor a disease or symptom severity scale index - the impact of disease is not systematically correlated with the disease severity as it is categorized (32) by health -care providers. Patient' or caregiver - 
based assessment can complement traditional clinical or biological evaluation, which could suggest that HRQOL could function as part of an early assessment or as part of disease monitoring.

Our findings were obtained in newly diagnosed and mild cases of asthma. Studies addressing established childhood asthma showed that the correlations between the clinical status and HRQOL scores are weak to moderate, even for disease-specific measures ( $29,30,31)$. A perception of dyspnea, although a crucial asthma symptom, might show interpersonal and intrapersonal variations (33). Although children with severe asthma tend to have a poorer quality of life than children with milder disease (34), parental and children's perception can be influenced by a number of factors. Consistent with findings in the G. Guyatt's study, in children aged 7-10, clinicians cannot rely on parental reports to provide insight into the child's HRQOL. This study also showed that the patient's assessment of change in symptoms were more closely related to HRQOL scores than parental assessment (20). A different effect provided by our study could be discussed in terms of early diagnosis and mild clinical presentation of the newly diagnosed cases.

Parental experience of their child's disease is very important for the diagnosis of asthma, and it would confirm the importance of screening programs addressed to parents, including emotional context of their functioning in relation to the child's symptoms. Parental HRQOL could be recommended as a valuable method of a more holistic assessment of disease impact on everyday functioning of child and their family. Both patient and parental perception are important in care strategies planning and implementation. Children are influenced by their parents and reduction of parental distress could be beneficial for child's family life and integration within 
society (35). In contrast an increasing parental stress could diminish compliance and adjustments to illness $(35,36)$.

The limitation of the study is its relatively small sample, although over 2000 randomly selected children were involved in the study. Despite the response rate being $80.8 \%$ (1822 children) during the first stage of study, fewer children responded to invitation for clinical evaluation (the response rates ranged from 25.6 to $70.8 \%$ depending the clinical group). Decreasing response rates should be considered as a limitations if longitudinal assessment would be considered. Final data collection (HRQOL) was completed on 29 newly diagnosed asthmatic children and 21 parents. Thus not all parents responded to HRQOL assessment and this should be also considered as the limitation of this study.

More complete data could be received if a multicenter study was conducted or if rural areas were included. Further studies are necessary to determine whether similar results could be found in other areas.

Other aspects should be considered, for example parental education or financial situation. Such conditions could be considered as possible mediators in a future research. Further studies could explain the role of social factors in the underdiagnosis of asthma in children.

Nevertheless the method used in our study provides pertinent data concerning the parental perception of patient disease and the questionnaire is a useful method to describe the impact of disease on parental quality of life. Pre and post-intervention HRQOL as an outcome measure could be implemented for evaluation education or coaching programs, and provide important information if strategies satisfy individual needs and health expectations. 
A general message provided by our study is that even unrecognized asthma (usually mild cases) has an important impact on every-day family functioning. What seems to be of importance is that asthma-related emotional changes were visible both in parents and in affected children. This observation could translate into a practically important result. The added value of the assessment of HRQOL extends beyond the diagnostic phase. It offers a better means of asthma management, such as for example family-tailored education and coaching. Therefore it is necessary to consider a multidisciplinary approach in the childhood asthma, even in mild cases, as ill children and their parents are subjects of care.

In conclusion, HRQOL newly recognized children with asthma and their parents doesn't correlate closely with clinical status. Patient and parental HRQOL should be considered as independent dimensions.

\section{References}

1. Pearce N, Ait-Khaled N, Beasley R, Mallor J, Keil U, Mitchell E, Robertson C and ISSAC Phase III Study Group. Thorax 2007; 62: 758-766

2. Gergen P, Mullally D, Evans R, National Survey of Prevalence of Asthma Among Children in the United States 1979 to 1980. Pediatrics. 1988, 8:1-7.

3. European Community Respiratory Health Survey. Variations in the prevalence of respiratory symptoms, self-reported asthma attacks and use of asthma medication in the European Community Respiratory Survey. Eur Respir J 1997; 10: 1795-1802.

4. Clark N, Brown R, Parker E, Robins T, Remick D, Philbert M Chilhood Asthma. Environ Health Perspect 1999; 107: 421-429. 
5. Asher MI, Montefort S, Bjorgsten B, Lai CK, Strachan DP, Weiland SK, Williams H. Worldwide time trends in the prevalence of symptoms of asthma, allergic rhinoconjuctivitis, and eczema in childhood: ISSAC Phases One and Three repeat multicountry cross-sectional surveys. Lancet 2006; 368: 733-743

6. Bjorksten B, Clayton T, Ellwod P, Steward A, Strachan D. Worldwide time trends for symptoms of rhinitis and rhinoconjuctivitis: Phase III of the International Study of Asthma and Allergies in Childhood. Pediatr Allergy Immunol 2008; 19: 110-124.

7. Brożek G, Zejda JE, Gebus M, Kepa K, Igielski M: Increase in the occurrence of allergic diseases in Polish children over past 15 years. Allergy 2008, Vol 63; suppl 88: 159 .

8. Gerald L, Redden D, Turner-Henson A, Feinstein R, Hemstreet M, Hains C, Brooks C, Erwin S, Bailey W. A Multi-stage Asthma Screening Procedures for Elementary Children. J Asthma 2002; 39 (1): 29-36.

9. Ben-Gashir M, Speed PT, Hay RJ. Quality of life and disease severity are correlated in children with atopic dermatitis. Br J Dermatol 2004; 150: 284-290. 10. Laforest L, Bousquet J, Pietri G, Kocevar VS, Yin D, Pacheco Y, Ganse EV. Quality of Life during Pollen Season in Patients with Seasonal Allergic Rhinitis with or without Asthma. Int Arch Allergy Immunol 2005, 136: 281-286.

11. Juniper EF, Guyatt GH, Ferrie PJ. et al Measuring quality of life in asthma. 1993, Am Rev Respir Dis, 147: 832-838

12. Marks GB, Dunn SM, Woolcock AJ A scale for measurement of quality of life in adults with asthma. J Clin Epidemiol 1992; 45: 461-472

13. Hyland ME, Finnis S, Irvine SH. A scale for assessing quality of life in adult asthma sufferers. J Psychosom Res 1991, 35: 99-110. 
14. Juniper EF. How Important is of Life in Pediatric Asthma? Ped Pulmon, 1997; suppl 15: 17-21.

15. Juniper EF, Wisniewski ME, Cox FM, Emmet AH, Nielsen KE, O’Byrne PM Relationship between quality of life and measures of clinical status in asthma: a factor analysis. Eur Respir J, 2004; 23: 287-291.

16. Townsend M, Feeny DH, Guyatt G, Furlong WJ, Seip AE, Dolovich J. Evaluation of burden of illness for pediatric asthmatic patients and their parents. Annals Allergy $1991 ; 67: 403-408$.

17. Skoner D. Outcome Measures in Childhood Asthma, Ped 2002; 109: 393-398.

18. Juniper EF, Guyatt GH, Feeny DH, Ferrie PJ, Grififith LE, Townsend M.

Measuring quality of life in children with asthma. Qual Life Res 1996; 5: 35-46.

19. Marks GB, Dunn SM, Woolcock AJ, A scale for the measurement of quality of life in adults with asthma. J Clin Epidemiol 1992; 45: 461-472

20. Guyatt G, Juniper E, Griffith L, Feeny D, Ferrie P. Children and Adult Perception of Childhood Asthma. Ped, 1997; 99(2): 165-168

21. Landis JR, Koch GG. The measurement of observer agreement for categorical data. Biometrics 1977;33:159-74.

22. Brożek G, Zejda JE: Increase in frequency of diagnosed allergic diseases in children - fact or artefact. Ped Pol 2004; 79: 385-392.

23. Zejda JE, Skiba M, Orawiev A, Dybowska T, Cimander B. Respiratory symptoms in children of Upper Silesia, Poland: cross-sectional study in two towns of different air pollution. Eur J Epidemiol 1996; 12: 115-120.

24. Leonardi GS, Houthuijs D, Nikiforov B, Volf J, Rudnai P, Zejda JE. Respiratory symptoms, bronchitis and asthma in children of Central and Eastern Europe. Eur Respir J 2002; 20: 890-898. 
25. Bateman ED, Hurd SS, Barnes PJ, Bousquet J, Drazen JM, Fitzgerald M, Gibson P, Ohta K, O'Byrne P, Petersen SE, Pizzichini E, Sullivan SD, Wenzel SE, Zar HJ. Global Strategy for Asthma Management and Prevention, Global Initiative for Asthma Management and Prevention: GINA executive summary. Eur Respir J 2008; 31: 143178. Available from: http://www.ginasthma.org

26.Brusasco V, Crapo R, Viegi G: ATS/ERS Task Force: Standardization of lung function testing - General considerations for lung function testing. Eur Respir J 2005; 26: 153-161.

27. Gerald L B, Grand R, Turner-Henson A, Hains C, Tang S, Feinstein A. Validation of a Multistage Asthma Case-Detection Procedure for Elementary School Children. Pediatrics 2004; 114: 459-468.

28. Callery P, Milnes L, Verduyn C, Coureil J. Qualitative study of young people's and parents' beliefs about childhood asthma. B J Gen Pract, 2003, 53; 185-190 29. Juniper EF, Guyatt GH, Ferrie PJ, Griffith LE. Measuring quality of life in asthma. Am Rev Respir Dis 1993; 147: 832-838.

30. Juniper EF, Norman GR, Cox FM, Roberts JN. Comparison of the standard gamble, rating scale, AQLQ and SF36 for measuring quality of life in adults. Eur Respir J, 2001; 18: 38-44.

31. Piquette CA, Clarckson L, Okamoto K, Kim JS, Rubin BK. Respiratory-related quality of life; Relation to pulmonary function, functional exercise capacity and sputum biophysical properties. J Aerosol Med, 2000: 13: 267-72 32. Patrick D, Erickson P. Health status and health policy. Quality of life in health care evaluation and resource allocation. Oxford University Press, 1993. 
33. Juniper EF, Wisniewski ME, Cox FM, Emmet AH, Nielsen KE, O’Byrne PM Relationship between quality of life and measures of clinical status in asthma: a factor analysis. Eur Respir J, 2004, 23: 287-291.

34. Martinez - Moragon E, Perpina M, Belloch A, de Diego A, Martinez-Frances ME. Asthma patients' perception of dyspnea during acute bronchoconstriction. Arch Bronchoneumol 2003; 39 (2): 67-73.

35. Trute B, Hiebert - Murphy D, Family adjustment to Childhood Developmental Disability : a measure of parent appraisal of family impacts. J Ped Psych; 2002; 27: $271-280$

36. Streisand R, Braniecki S, Tercyak KP, Childhood Illness - Related Parenting Stress: The Pediatric Inventory for Parents. J Ped Psych, 2001; 26 (3): 155-162.

Acknowledgements

The respiratory survey was sponsored by scientific grant from State Committee for Scientific Research (Polish Ministry of Scientific Research and Information Technology). The authors are grateful to Professor Elizabeth Juniper for her kind permission to use the PAQLQ. 


\section{Quality of Life Protocol in the Early Asthma Diagnosis in Children}

Małgorzata Farnik MD PhD'1, Władysław Pierzchała MD PhD¹, Grzegorz Brożek MD $\mathrm{PhD}^{2}$, Jan E. Zejda MD PhD², Michał Skrzypek $\mathrm{MS}^{3}$.

1 Department of Pneumonology, Medical University of Silesia, Katowice, Poland

2 Department of Epidemiology, Medical University of Silesia, Katowice, Poland

${ }^{3}$ Department of Biostatistics, Faculty of Public Health, Medical University of Silesia, Bytom, Poland

Correspondence: Małgorzata Farnik MD PhD

Department of Pneumonology

Medical University of Silesia

Ul. Medyków 14

40-752 Katowice, Poland

tel/fax. 48-32-252-38-31

e-mail pneumo@sum.edu.pl

Key words: asthma, pediatrics, health-related quality of life, early diagnosis

Abbreviated title: QOL in early asthma diagnosis 


\section{Summary}

Introduction. Asthma is considered an important problem in children and influences on their everyday functioning. This study was concerned with the impact of asthma on the health related quality of life (HRQOL) of newly recognized pediatric asthma patients and their parents.

Methods. Patients were identified by a general respiratory survey. HRQOL was assessed by self-report measures: disease-specific questionnaire for children, the Pediatric Asthma Quality of Life Questionnaire (PAQLQ) and generic for parents - the Quality of Life in Child's Chronic Disease Questionnaire (QLCCDQ).

Results. 29 newly diagnosed asthmatic children aged $7-11$ years (mean age $=8.6$ $S D=1.4$ ) were identified from a population-based respiratory health survey. The most impaired HRQOL domains differed in parents and children. In children, activity limitation was the most impaired; however in parents, emotional functioning and symptom perception domains were most impaired. There were no statistically significant differences in patient and parental HRQOL observed in either the group where symptoms were present or in the group were symptoms were not present. Baseline spirometry showed correlation for $\mathrm{FEV}_{1} \% \mathrm{FVC}$ and PAQLQ symptom domain $(r=0.42)$. Exercise test showed correlations between $\mathrm{FEV}_{1} \% \mathrm{FVC}$ and QLCCDQ emotional domain ( $r=0.7)$; MEF25-75\% and family roles $(r=0.81)$, as well as social functioning $(r=0.81)$. Activity limitation on the PAQLQ correlated with the MEF 25-75\% ( $r=0.44)$ and PEF $(r=0.55)$.

Conclusions. HRQOL newly recognized children with asthma and their parents does not correlate closely with clinical status. Patient and parental HRQOL should be considered as independent dimensions. 


\section{Introduction}

The results of International Study of Asthma and Allergies in Childhood (ISAAC) have shown that childhood asthma represents an important public health problem worldwide (1). Previously increasing prevalence of childhood asthma, well documented in many countries $(2,3,4)$ can be observed in Eastern Europe over the years 1994-2007 $(1,5,6,7)$. The magnitude of the problem could be underestimated because of underdiagnosis (8). The resulting public health burden justifies the development and implementation of population-based measures. Asthma not only has a profound impact on public health, but also on the everyday lives of individual patients.

A patients' burden of chronic illness cannot be assessed by disease severity; it requires measurement reflecting personal perception. There is an increasing interest in activity limitation, emotional functioning, impact on every-day life due to disease and the implementation of health-related quality of life (HRQOL) questionnaires. Deterioration of HRQOL has been described in children and adults with allergic diseases, such as eczema (9) and rhinitis (10). HRQOL assessment highlights important and troublesome problems experienced by patients with asthma $(11,12,13)$. Children with asthma suffer from activity limitation, emotional problems such frustration, fear and irritation (14). Although HRQOL does not correlate closely with asthma control, it is known that children with very severe asthma tend to have a poorer quality of life than those suffering from mild or moderate condition (15). Parents of children with asthma could be limited in normal daily activities and experience emotional distress (16). 
Clinical experience shows that a caregiver's HRQOL and their perception of a child's symptoms are important in diagnosis and control of established asthma (17). Less is known about the potential impact of disease on HRQOL in newly recognized children with asthma which were found as the result of screening programs. It remains unknown to what extent such a procedure could be helpful in both early assessment of asthma and its impact on patients' and parental quality of life.

We implemented the HRQOL protocol in examination of newly identified pediatric asthma patients and their families, participants of a large population-based respiratory health survey. The goal of the study was to determine the impact of asthma on the health related quality of life of newly recognized pediatric asthma patients and their parents. 


\title{
Materials and Methods
}

\begin{abstract}
A large population-based respiratory health survey resulted in identification of new pediatric asthma patients. All newly identified children with asthma aged 7-11 years and their parents were involved in HRQOL study. A disease-specific quality-oflife questionnaire was used for the children and a generic questionnaire for parents. Clinical status data was obtained prior to an HRQOL assessment.
\end{abstract}

\section{$\underline{\text { Participants }}$}

Children were recruited using cluster sampling during a respiratory health survey study in primary schools in Katowice (Southern Poland). 2254 children grades I-IV, in 7 out of 51 schools in the town were invited $(17.0 \%$ of the town population in the defined age group) for respiratory questionnaire assessment. This was a validated questionnaire, used in The Central European Study of Air Pollution and Respiratory Health (CESAR) [Table 1] and other surveys $(22,23,24)$. The response rate was $80.8 \%$ (1822 children). Study design is shown in Fig. 1 Figure 1. Study design

Based on the questionnaire results children were categorized into three mutually exclusive groups: "Asthma" (5.4\% - diagnosis of asthma established before the survey); "Respiratory Symptoms" (27.9\% - no previous diagnosis of asthma and one or more symptoms of persistent cough excluding infections, chest wheezing, dyspnea during the last 12 months), "No Respiratory Symptoms" (66.7\% - no previous diagnosis of asthma and no respiratory symptoms during the last 12 months). 
[Table 1] - Respiratory Questionnaire (examples of questions)

Clinical evaluation was proposed to all children in "Asthma"- group and 60\% samples from groups "Respiratory Symptoms" and "No Respiratory Symptoms", with the response rates: $41.4 \%, 70.8 \%$ and $25.6 \%$, respectively. A certified pulmonologist (Department of Pneumonology, Medical University of Silesia) examined 415 children. The physician was blind to the results of the questionnaire. Diagnostic procedures were based on GINA guidelines for childhood asthma (25). Forced expiratory volumes were measured according to ERS/ATS recommendations (26). Spirometry was considered normal if $\mathrm{FEV}_{1}, \mathrm{FVC}$ and PEF values exceeded $80 \%$ predicted and if $\mathrm{FEV}_{1} \% \mathrm{FVC}$ ratio was greater than $75 \%$. If actual values presented predicted values, a further challenge test of bronchial hyperresponsiveness, described by Gerald et al (27), was used. A sub-maximal exercise test (stepping up and down on a single step with metronome rhythm) was followed by spirometry obtained at minutes 3,5 and 10 after exercise. An $\mathrm{FEV}_{1}$ decrease greater than $15 \%$ was considered positive for asthma. Medical records were provided; additional skin allergy tests and a chest radiograph were carried out, where needed.

Clinical assessment of 415 children confirmed 33 previously known and revealed 29 new cases of asthma. All newly identified children with asthma and their parents were invited to HRQOL assessment.

\section{$\underline{\text { HRQOL measurements }}$}

Quality of life questionnaires were completed by children with asthma at school in the patient's normal psychosocial environment. Parents of newly diagnosed children 
with asthma completed HRQOL questionnaire as well at school, before the diagnosis was communicated.

The Paediatric Asthma Quality of Life Questionnaire

The Paediatric Asthma Quality of Life Questionnaire (PAQLQ), dedicated to children aged 7-17 years old, was used as a well-validated and reliable measure of HRQOL in childhood asthma. The questionnaire consists of 23 questions, covering symptoms, emotional function and limitations of activity. Children were asked to recall their experiences over the previous week and responded on a 7 - point scale ( 7 - no impairment, 1 - severe impairment). Patients completed the questionnaire with physicians experienced in HRQOL research. Interviewer-administration was used to avoid parental interference with patient responses.

\section{Quality of Life in a Child's Chronic Disease Questionnaire}

An accompanying questionnaire for parents was devised by the authors, based on standard procedure - starting from initial questions (identification of needs, definition of operational objectives), tool development, pretesting and final version (format, wording) preparation, pilot testing and validation of the questionnaire $(19,20)$. Quality of Life in a Child's Chronic Disease Questionnaire (QLCCDQ) is concerned with daily problems and limitations that concern parents of children with chronic disease. QLCCDQ is a self - report measure of parent's HRQOL, only symptoms subscale is parent-proxy measure. Most questions concern parents' functioning, only the symptom subscale reflects parents' perception of symptoms presence.

QLCCDQ consisted of 15 questions and covered patient-perceived symptoms (e.g Did the child show any worrying symptoms?), emotions (e.g. Have you experienced anxiety because of your child's health problems?) and activity limitation in roles function: social (e.g. Please indicate how much you have been limited by your child's 
disease in listed activities in past two weeks: going to church, cinema, visiting friends), family roles (e.g. Do you struggle to find time to spend with other family members (spouse, another child) because of your child' disease?) and occupational (e.g. Please indicate how much you have been limited by your child's disease in work related activities in past two weeks).

The questionnaire required circling a choice from seven responses devised for each question, from 1 (most bothered or limited) to 7 (not bothered or limited). All questions concerned parental HRQOL of previous two-week period.

\section{Psychometric data}

\section{PAQLQ}

The instrument is reproducible $(\mathrm{ICC}=0.95)$ and has shown ability to detect HRQOL changes as a result of treatment $(p<0.001)$. Estimation of reliability in stable asthma has shown that it was consistent throughout all domains (ICC: 0.84-0.93).

\section{QLCCDQ}

Reproducibility based on the weighted kappa coefficients showed an expected level of agreement. Values of weighed kappa coefficients suggested almost perfect agreement $(0.81-1.0)$ in case of 8 questions, substantial $(0.61-0.8)$ for 5 questions and moderate $(0.41-0.6)$ for 2 questions. The reliability of the defined subscales was evaluated by means of Cronbach's $\alpha$. The values of $\alpha$ ranged between 0.74 and 0.94: symptoms: 0.93 , emotions: 0.91 , family roles: 0.77 , social roles: 0.79 , occupational roles: 0.92 and activity limitations in roles function: 0.91 .

\section{$\underline{\text { Child's school grades and absenteeism }}$}


Supplementary data such as school grades and absenteeism (missed school days due to asthma symptoms) were provided to determine if there were relationships between reported HRQOL and child's functioning at school. It could be expected that untreated asthma has an impact on school functioning resulting in children lower school grades and missed school days. Polish school grades assessment is based on 6- point scale: from 1 (Fail) to 6 (Excellent).

The study protocol was approved by the ethics committee (Medical University of Silesia). Each child admitted to the study had the written consent of a parent.

\section{Statistical analyses}

The statistical analysis was performed using standard procedures available in the Statistica 7.1 package and SAS, version 9.2 (SAS Institute Inc., Gary, NC). Normality of distributions of continuous variables was analyzed using the ShapiroWilk test. Statistical significance of differences between continuous variables was analyzed by t-Student test and ANOVA while for data with non-normal distribution, $U$ Mann-Whitney and Kruskal-Wallis tests were used. Differences between categorical variables were examined by the chi-square test. Strength of associations, due to nonnormal distributions, were measured by Spearman's rank correlation coefficient.

The statistical inferences were based on confidence levels $p<0.05$. If multiple comparisons were used statistical significance was lowered according to the Bonferroni adjustment. 
Variables included patient and parental HRQOL assessment, symptoms over the previous 12 months, baseline and exercise spirometry, school grades and absenteeism.

Correlations between child's and parental HRQOL scores were analyzed. Relations between clinical status (symptoms, lung function tests) and HRQOL were examined, as well as links between educational markers (school grades and absenteeism) and patient HRQOL scores. Gender and age effects on HRQOL were also analyzed. 


\section{Results}

\section{$\underline{\text { Participating children }}$}

In children with newly diagnosed asthma (mean age $=8.6$ years; $S D=1.4$ years), classified according to GINA protocol, the disease was either episodic (20 children: 6 boys and 14 girls) or chronic mild ( 9 children: 4 boys and 5 girls). New diagnoses of asthma were made in $10.9 \%$ of children from the "Respiratory Symptoms" group and in $3.6 \%$ of children from the "No Respiratory Symptoms" group. All 29 newly diagnosed children with asthma and 21 parents responded to the subsequent HRQOL questionnaire.

[Table 2 ] - Demographic and asthma severity

\section{Patient and parental HRQOL}

HRQOL was assessed for each domain, the subscale mean ranging from 1.0 to 7.0. Lower HRQOL scores reflected greater impact of disease on every-day functioning. Children's most affected HRQOL domain was limitation of activity. Parents' impairment was most severe in emotional functioning and perceived symptoms, while less impaired domains included social functioning. Domains and overall HRQOL scores for children and parents appear in the table 3.

[Table 3]. HRQOL - domains and overall scores

Statistically important positive correlations were observed between all PAQLQ subscales, as well as most QLCCDQ subscales [ Table 4]. QLCCDQ symptom perception subscale correlated only with parental emotional functioning, while 
correlations were found between most subscales describing roles functioning.

Bonferroni correction suggested significance level was $p<0.0001$.

[Table 4]. PAQLQ and QLCCDLQ subscales Spearman's correlations

\section{$\underline{H R Q O L}$ measures and clinical assessment}

Symptoms

The differences of mean QLCCDQ and PAQLQ subscales scores between groups of symptomatic and nonsymptomatic children over in the past 12 months were analyzed. Both for QLCCDQ and PAQLQ the differences in scores did not reach statistical significance. Bonferroni correction suggested significance level was $p<$ 0.01 .

\section{Lung function tests}

Statistically important correlations were found between HRQOL and spirometry. Challenge tests were done in 23 of the 29 children -3 children did not obtain reproducible spirometry after exercise, 2 children were wheezing before exercise commenced, 1 child refused to cooperate. Baseline FEV $\%$ FVC and symptom domain in children showed a correlation $(r=0.42)$, while exercise value $\mathrm{FEV}_{1} \% \mathrm{FVC}$ correlated with emotional domain ( $\left.r=0.7\right)$. MEF25-75\% correlated with QLCCDQ family roles $(r=0.81)$ and social functioning $(r=0.81)$. PAQLQ activity limitation was correlated with MEF 25-75\% $(r=0.44)$ and PEF $(r=0.55)$, both obtained during the exercise test. All exercise test correlations were based on spirometry results collected 10 minutes after the challenge test [Table 5].

[ Table 5]. HRQOL scores and lung function - Spearmans' correlations 


\section{$\underline{\text { School grades and absenteeism }}$}

Higher scores on the symptoms subscale of PAQLQ correlated with better school grades $(r=0.64 ; p=0.02)$ and fewer missed school days $(r=-0.53 ; p=0.02)$. No correlations were observed in parents' HRQOL and missed school days or school marks.

\section{Patient gender and age}

Females showed a significantly lower symptom score in comparison to males $(p=0.01)$, however this effect was not translated into total PAQLQ scores nor into parents' HRQOL.

The relationship between HRQOL scores and age were not statistically significant. 


\section{Discussion}

Our study focused on the implementation of the HRQOL protocol in early diagnosis of childhood asthma. The analysis involved data obtained in children with newly diagnosed asthma and in their parents. Since asthma cases were identified through population-based screening, it is not surprising that all previously undiagnosed cases represented either episodic or mild asthma - children with more advanced disease had been diagnosed on regular basis, via pediatric health care. An important finding of our study is that the profile of HRQOL impairment is different in children with asthma and in their parents. Not only did PAQLQ and QLCCDQ scores not correlate but - in addition - the highest impaired domains differed between children and parents. Symptom perception and emotional functioning appeared to be the most affected HRQOL domains in parents, while activity limitation was the most impaired domain in children. Some parents might limit their child's activity, due to emotional distress caused by anticipation of symptoms, and such behavior could explain the lack of correlation between emotion domain QLCCDQ and symptom or activity limitation domains in PAQLQ.

It is of interest that parental HRQOL pattern is in line with the published qualitative evidence that parents tend to perceive symptoms of asthma in a child as intermittent acute episodes rather than a chronic condition (28). Such interpretation could explain the lack of diagnosis of episodic or mild asthma in our study. It cannot be excluded that such a presentation is not entirely neglected by parents, but treated by them as an annoying condition and not overt disease. Our finding could have some implication as far as screening is concerned - occurrence of symptoms is necessary 
but does not have to be a sufficient cause to seek medical advice. Other important factors include individual experience, perception and emotions.

The relationships between clinical indices and HRQOL were analyzed in many studies as an important observation for clinicians $(29,30,31,32)$. Our study has shown that there were no statistically significant differences in both patient and parental HRQOL scores between symptomatic and asymptomatic groups. However some of analyzed relationships were suggested as significant; before the significance level was lowered by applying Bonferroni correction to $p<0.01$. As the example compared to parents of asymptomatic children, parents of children complaining of wheezing had a lower $(p=0.02)$ mean score in emotional function and symptom domain. As well the group of children possessing dyspnea showed a lower mean parental emotional function score $(p=0.04)$ than in asymptomatic children. In children an emotional function score was lower in the group of children complaining of cough in the past 12 months $(p=0.01)$ as compared to asymptomatic children.

Correlations were observed for exercise lung function and QLCCDQ domains, such as emotional, family and social functions. Worthy to note, that the correlations between children's lung function and parental HRQOL domains were stronger than analogous correlations concerning HRQOL in children. The most impaired parent HRQOL domain - emotions, significantly correlated with FEV $1 \%$ FVC. Activity limitation, the most impaired patient domain, correlated with PEF and MEF 25-75. Even if the correlations between clinical indices and HRQOL scores were observed only for few variables it should not determine a limitation of HRQOL protocol in early diagnosis. As HRQOL is not a diagnostic scale nor a disease or symptom severity scale index - the impact of disease is not systematically correlated with the disease severity as it is categorized (32) by health -care providers. Patient' or caregiver - 
based assessment can complement traditional clinical or biological evaluation, which could suggest that HRQOL could function as part of an early assessment or as part of disease monitoring.

Our findings were obtained in newly diagnosed and mild cases of asthma. Studies addressing established childhood asthma showed that the correlations between the clinical status and HRQOL scores are weak to moderate, even for disease-specific measures (29, 30, 31). A perception of dyspnea, although a crucial asthma symptom, might show interpersonal and intrapersonal variations (33). Although children with severe asthma tend to have a poorer quality of life than children with milder disease (34), parental and children's perception can be influenced by a number of factors. Consistent with findings in the G. Guyatt's study, in children aged 7-10, clinicians cannot rely on parental reports to provide insight into the child's HRQOL. This study also showed that the patient's assessment of change in symptoms were more closely related to HRQOL scores than parental assessment (20). A different effect provided by our study could be discussed in terms of early diagnosis and mild clinical presentation of the newly diagnosed cases.

Parental experience of their child's disease is very important for the diagnosis of asthma, and it would confirm the importance of screening programs addressed to parents, including emotional context of their functioning in relation to the child's symptoms. Parental HRQOL could be recommended as a valuable method of a more holistic assessment of disease impact on everyday functioning of child and their family. Both patient and parental perception are important in care strategies planning and implementation. Children are influenced by their parents and reduction of parental distress could be beneficial for child's family life and integration within 
society (35). In contrast an increasing parental stress could diminish compliance and adjustments to illness $(35,36)$.

The limitation of the study is its relatively small sample, although over 2000 randomly selected children were involved in the study. Despite the response rate being $80.8 \%$ (1822 children) during the first stage of study, fewer children responded to invitation for clinical evaluation (the response rates ranged from 25.6 to $70.8 \%$ depending the clinical group). Decreasing response rates should be considered as a limitations if longitudinal assessment would be considered. Final data collection (HRQOL) was completed on 29 newly diagnosed asthmatic children and 21 parents. Thus not all parents responded to HRQOL assessment and this should be also considered as the limitation of this study.

More complete data could be received if a multicenter study was conducted or if rural areas were included. Further studies are necessary to determine whether similar results could be found in other areas.

Other aspects should be considered, for example parental education or financial situation. Such conditions could be considered as possible mediators in a future research. Further studies could explain the role of social factors in the underdiagnosis of asthma in children.

Nevertheless the method used in our study provides pertinent data concerning the parental perception of patient disease and the questionnaire is a useful method to describe the impact of disease on parental quality of life. Pre and post-intervention HRQOL as an outcome measure could be implemented for evaluation education or coaching programs, and provide important information if strategies satisfy individual needs and health expectations. 
A general message provided by our study is that even unrecognized asthma (usually mild cases) has an important impact on every-day family functioning. What seems to be of importance is that asthma-related emotional changes were visible both in parents and in affected children. This observation could translate into a practically important result. The added value of the assessment of HRQOL extends beyond the diagnostic phase. It offers a better means of asthma management, such as for example family-tailored education and coaching. Therefore it is necessary to consider a multidisciplinary approach in the childhood asthma, even in mild cases, as ill children and their parents are subjects of care.

In conclusion, HRQOL newly recognized children with asthma and their parents doesn't correlate closely with clinical status. Patient and parental HRQOL should be considered as independent dimensions.

\section{References}

1. Pearce N, Ait-Khaled N, Beasley R, Mallor J, Keil U, Mitchell E, Robertson C and ISSAC Phase III Study Group. Thorax 2007; 62: 758-766

2. Gergen P, Mullally D, Evans R, National Survey of Prevalence of Asthma Among Children in the United States 1979 to 1980. Pediatrics. 1988, 8:1-7.

3. European Community Respiratory Health Survey. Variations in the prevalence of respiratory symptoms, self-reported asthma attacks and use of asthma medication in the European Community Respiratory Survey. Eur Respir J 1997; 10: 1795-1802.

4. Clark N, Brown R, Parker E, Robins T, Remick D, Philbert M Chilhood Asthma. Environ Health Perspect 1999; 107: 421-429. 
5. Asher MI, Montefort S, Bjorgsten B, Lai CK, Strachan DP, Weiland SK, Williams H. Worldwide time trends in the prevalence of symptoms of asthma, allergic rhinoconjuctivitis, and eczema in childhood: ISSAC Phases One and Three repeat multicountry cross-sectional surveys. Lancet 2006; 368: 733-743

6. Bjorksten B, Clayton T, Ellwod P, Steward A, Strachan D. Worldwide time trends for symptoms of rhinitis and rhinoconjuctivitis: Phase III of the International Study of Asthma and Allergies in Childhood. Pediatr Allergy Immunol 2008; 19: 110-124.

7. Brożek G, Zejda JE, Gebus M, Kepa K, Igielski M: Increase in the occurrence of allergic diseases in Polish children over past 15 years. Allergy 2008, Vol 63; suppl 88: 159 .

8. Gerald L, Redden D, Turner-Henson A, Feinstein R, Hemstreet M, Hains C, Brooks C, Erwin S, Bailey W. A Multi-stage Asthma Screening Procedures for Elementary Children. J Asthma 2002; 39 (1): 29-36.

9. Ben-Gashir M, Speed PT, Hay RJ. Quality of life and disease severity are correlated in children with atopic dermatitis. Br J Dermatol 2004; 150: 284-290. 10. Laforest L, Bousquet J, Pietri G, Kocevar VS, Yin D, Pacheco Y, Ganse EV. Quality of Life during Pollen Season in Patients with Seasonal Allergic Rhinitis with or without Asthma. Int Arch Allergy Immunol 2005, 136: 281-286.

11. Juniper EF, Guyatt GH, Ferrie PJ. et al Measuring quality of life in asthma. 1993, Am Rev Respir Dis, 147: 832-838

12. Marks GB, Dunn SM, Woolcock AJ A scale for measurement of quality of life in adults with asthma. J Clin Epidemiol 1992; 45: 461-472

13. Hyland ME, Finnis S, Irvine SH. A scale for assessing quality of life in adult asthma sufferers. J Psychosom Res 1991, 35: 99-110. 
14. Juniper EF. How Important is of Life in Pediatric Asthma? Ped Pulmon, 1997; suppl 15: 17-21.

15. Juniper EF, Wisniewski ME, Cox FM, Emmet AH, Nielsen KE, O’Byrne PM Relationship between quality of life and measures of clinical status in asthma: a factor analysis. Eur Respir J, 2004; 23: 287-291.

16. Townsend M, Feeny DH, Guyatt G, Furlong WJ, Seip AE, Dolovich J. Evaluation of burden of illness for pediatric asthmatic patients and their parents. Annals Allergy $1991 ; 67: 403-408$.

17. Skoner D. Outcome Measures in Childhood Asthma, Ped 2002; 109: 393-398. 18. Juniper EF, Guyatt GH, Feeny DH, Ferrie PJ, Grififith LE, Townsend M. Measuring quality of life in children with asthma. Qual Life Res 1996; 5: 35-46. 19. Marks GB, Dunn SM, Woolcock AJ, A scale for the measurement of quality of life in adults with asthma. J Clin Epidemiol 1992; 45: 461-472

20. Guyatt G, Juniper E, Griffith L, Feeny D, Ferrie P. Children and Adult Perception of Childhood Asthma. Ped, 1997; 99(2): 165-168 21. Landis JR, Koch GG. The measurement of observer agreement for categorical data. Biometrics 1977;33:159-74.

22. Brożek G, Zejda JE: Increase in frequency of diagnosed allergic diseases in children - fact or artefact. Ped Pol 2004; 79: 385-392.

23. Zejda JE, Skiba M, Orawiev A, Dybowska T, Cimander B. Respiratory symptoms in children of Upper Silesia, Poland: cross-sectional study in two towns of different air pollution. Eur J Epidemiol 1996; 12: 115-120.

24. Leonardi GS, Houthuijs D, Nikiforov B, Volf J, Rudnai P, Zejda JE. Respiratory symptoms, bronchitis and asthma in children of Central and Eastern Europe. Eur Respir J 2002; 20: 890-898. 
25. Bateman ED, Hurd SS, Barnes PJ, Bousquet J, Drazen JM, Fitzgerald M, Gibson P, Ohta K, O'Byrne P, Petersen SE, Pizzichini E, Sullivan SD, Wenzel SE, Zar HJ. Global Strategy for Asthma Management and Prevention, Global Initiative for Asthma Management and Prevention: GINA executive summary. Eur Respir J 2008; 31: 143178. Available from: http://www.ginasthma.org

26.Brusasco V, Crapo R, Viegi G: ATS/ERS Task Force: Standardization of lung function testing - General considerations for lung function testing. Eur Respir J 2005; 26: 153-161.

27. Gerald L B, Grand R, Turner-Henson A, Hains C, Tang S, Feinstein A. Validation of a Multistage Asthma Case-Detection Procedure for Elementary School Children. Pediatrics 2004; 114: 459-468.

28. Callery P, Milnes L, Verduyn C, Coureil J. Qualitative study of young people's and parents' beliefs about childhood asthma. B J Gen Pract, 2003, 53; 185-190 29. Juniper EF, Guyatt GH, Ferrie PJ, Griffith LE. Measuring quality of life in asthma. Am Rev Respir Dis 1993; 147: 832-838.

30. Juniper EF, Norman GR, Cox FM, Roberts JN. Comparison of the standard gamble, rating scale, AQLQ and SF36 for measuring quality of life in adults. Eur Respir J, 2001; 18: 38-44.

31. Piquette CA, Clarckson L, Okamoto K, Kim JS, Rubin BK. Respiratory-related quality of life; Relation to pulmonary function, functional exercise capacity and sputum biophysical properties. J Aerosol Med, 2000: 13: 267-72 32. Patrick D, Erickson P. Health status and health policy. Quality of life in health care evaluation and resource allocation. Oxford University Press, 1993. 
33. Juniper EF, Wisniewski ME, Cox FM, Emmet AH, Nielsen KE, O’Byrne PM Relationship between quality of life and measures of clinical status in asthma: a factor analysis. Eur Respir J, 2004, 23: 287-291.

34. Martinez - Moragon E, Perpina M, Belloch A, de Diego A, Martinez-Frances ME. Asthma patients' perception of dyspnea during acute bronchoconstriction. Arch Bronchoneumol 2003; 39 (2): 67-73.

35. Trute B, Hiebert - Murphy D, Family adjustment to Childhood Developmental Disability : a measure of parent appraisal of family impacts. J Ped Psych; 2002; 27: $271-280$

36. Streisand R, Braniecki S, Tercyak KP, Childhood Illness - Related Parenting Stress: The Pediatric Inventory for Parents. J Ped Psych, 2001; 26 (3): 155-162.

Acknowledgements

The respiratory survey was sponsored by scientific grant from State Committee for Scientific Research (Polish Ministry of Scientific Research and Information Technology). The authors are grateful to Professor Elizabeth Juniper for her kind permission to use the PAQLQ. 
Figure 1. Study design

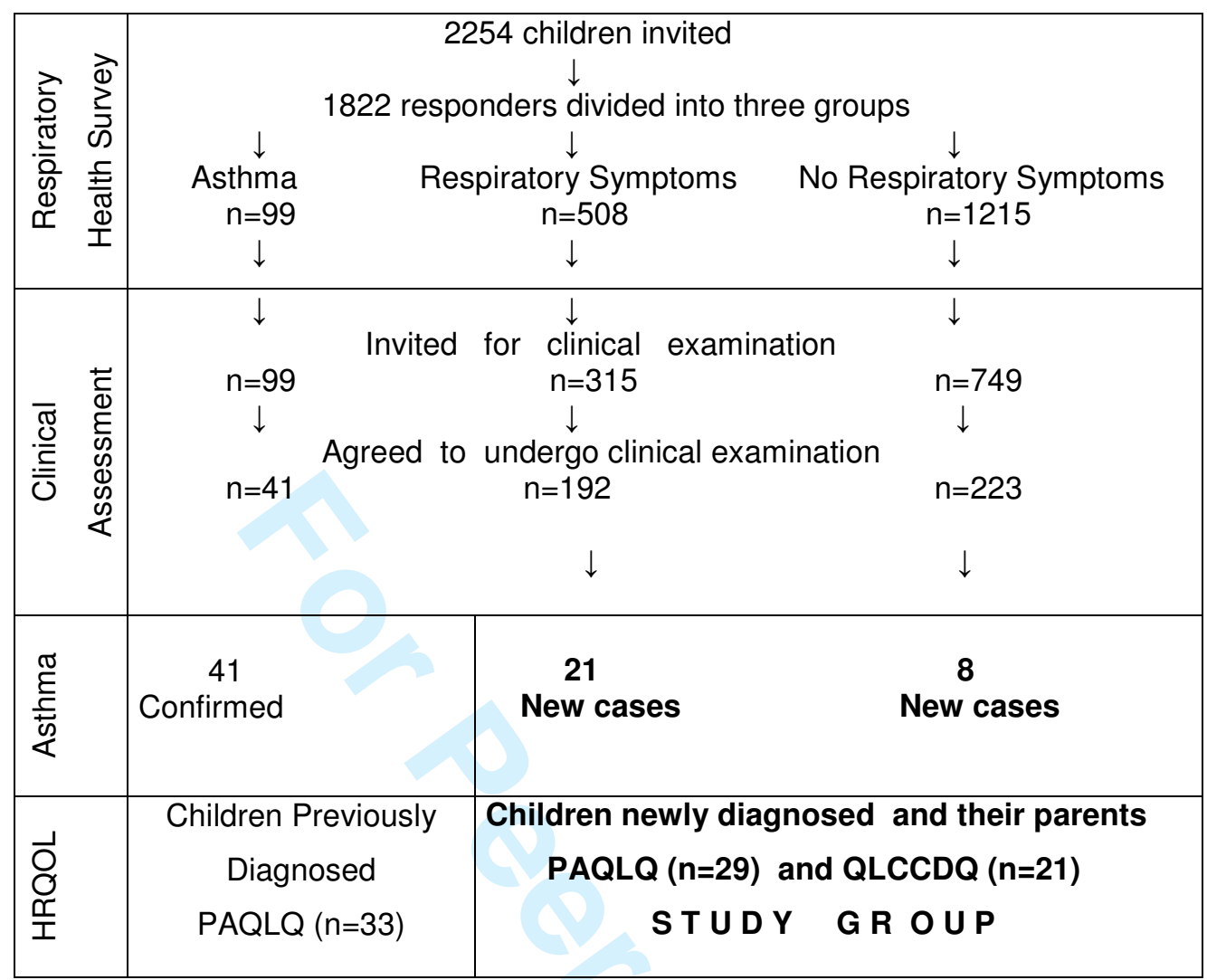


Table 1. Respiratory Questionnaire

\begin{tabular}{|c|c|c|}
\hline Symptom & Question & Clinical variables \\
\hline \multirow[t]{2}{*}{ Wheezing } & Has the child ever had chest wheezing & \\
\hline & Has the child had chest wheezing past 12 months & $\sqrt{ }$ \\
\hline \multirow[t]{2}{*}{ Dyspnea } & Has the child ever had attack of dyspnea & \\
\hline & $\begin{array}{l}\text { Has the child had attack of dyspnea during last } 12 \\
\text { months }\end{array}$ & $\sqrt{ }$ \\
\hline Dry cough & $\begin{array}{l}\text { In past } 12 \text { months has the child had a dry cough at } \\
\text { night, apart from a cough associated with a cold or } \\
\text { chest infection }\end{array}$ & $\sqrt{ }$ \\
\hline Morning cough & $\begin{array}{l}\text { Has the child had a morning cough during autumn } \\
\text { and winter }\end{array}$ & $\sqrt{ }$ \\
\hline Chronic cough & $\begin{array}{l}\text { Has the child coughed on most days for a minimum } \\
\text { of three months yearly for two years }\end{array}$ & $\sqrt{ }$ \\
\hline $\begin{array}{l}\text { Exercise induced } \\
\text { symptoms }\end{array}$ & $\begin{array}{l}\text { Has the child had exercise-induced wheezing, } \\
\text { dyspnea or cough in the last } 12 \text { months }\end{array}$ & $\sqrt{ }$ \\
\hline & Has the child ever had recognized obstructive & \\
\hline Previous diagnosis & $\begin{array}{l}\text { bronchitis, bronchial obstruction or asthma by } \\
\text { physician }\end{array}$ & \\
\hline
\end{tabular}


Table 2. Demographic and asthma severity

\begin{tabular}{|c|c|c|c|c|}
\hline & \multicolumn{2}{|c|}{ Males } & \multicolumn{2}{|c|}{ Females } \\
\hline & Number & Percent & Number & percent \\
\hline \multicolumn{5}{|l|}{ GINA classification } \\
\hline I & 6 & 60 & 14 & 73,68 \\
\hline II & 4 & 40 & 5 & 26,32 \\
\hline Total & 10 & 34,5 & 19 & 65,5 \\
\hline \multicolumn{5}{|l|}{ Age } \\
\hline Average (SD) & \multicolumn{2}{|c|}{$8,6(1,4)$} & \multicolumn{2}{|c|}{$8,9(1,0)$} \\
\hline
\end{tabular}


Table 3. HRQOL - domains and overall scores

\begin{tabular}{lrrrr}
\hline & Mean & SD & Min & Max \\
\hline $\begin{array}{l}\text { QLCCDQ domain } \\
\text { Role functioning }\end{array}$ & & & & \\
$\quad$ Family & 5.9 & 0.8 & 4.0 & 7.0 \\
$\quad$ Social & 6.1 & 0.7 & 4.3 & 7.0 \\
$\quad$ Occupational & 5.7 & 1.0 & 3.7 & 7.0 \\
Roles activity & 5.8 & 0.8 & 4.2 & 7.0 \\
limitations & 4.0 & 1.1 & 2.0 & 6.3 \\
Symptoms & 4.1 & 0.7 & 3.7 & 6.2 \\
Emotions & 81.7 & 10 & 66 & 98 \\
\hline Overall points & 5.4 & 0.7 & 4.4 & 6.5 \\
Overall mean & & & & \\
PAQLQ domain & 5.4 & 1.2 & 2.1 & 7.0 \\
Symptoms & 4.7 & 1.1 & 1.4 & 7.0 \\
Activity limitation & 5.9 & 1.3 & 1.4 & 7.0 \\
Emotions & 126.8 & 24,98 & 41 & 161 \\
\hline Overall points & 5.5 & 1.1 & 1.8 & 7.0 \\
\hline Overall mean & & & &
\end{tabular}


Table 4. QLCCDQ and PAQLQ subscales Spearmans' correlations

\begin{tabular}{|c|c|c|c|c|c|c|}
\hline & $\begin{array}{l}\text { Family } \\
\text { roles }\end{array}$ & Social roles & $\begin{array}{l}\text { Occupation } \\
\text { al roles }\end{array}$ & $\begin{array}{l}\text { Activity } \\
\text { limitation }\end{array}$ & $\begin{array}{l}\text { Symptoms } \\
\text { perception }\end{array}$ & Emotions \\
\hline \multicolumn{7}{|l|}{$\begin{array}{l}\text { Domain } 1 \\
\text { QLCCDQ }\end{array}$} \\
\hline Family roles & - & & & & & \\
\hline Social roles & $\begin{array}{l}0.7 \\
(0.0001)\end{array}$ & - & & & & \\
\hline $\begin{array}{l}\text { Occupational } \\
\text { Roles }\end{array}$ & $\begin{array}{l}0.6 \\
(0.007)\end{array}$ & $\begin{array}{l}0.6 \\
(0.007)\end{array}$ & - & & & \\
\hline $\begin{array}{l}\text { Roles activity } \\
\text { limitation }\end{array}$ & $\begin{array}{l}0.8 \\
(0.0001)\end{array}$ & $\begin{array}{l}0.8 \\
(0.0001)\end{array}$ & $\begin{array}{l}0.9 \\
(0.0001)\end{array}$ & - & & \\
\hline $\begin{array}{l}\text { Symptoms } \\
\text { perception }\end{array}$ & $\begin{array}{l}0.07 \\
(0.7)\end{array}$ & $\begin{array}{l}-0.005 \\
(0.9)\end{array}$ & $\begin{array}{l}0.4 \\
(0.08)\end{array}$ & $\begin{array}{l}0.2 \\
(0.31)\end{array}$ & - & \\
\hline Emotions & $\begin{array}{l}0.2 \\
(0.4)\end{array}$ & $\begin{array}{l}0.2 \\
(0.3)\end{array}$ & $\begin{array}{l}0.4 \\
(0.05)\end{array}$ & $\begin{array}{l}0.4 \\
(0.09)\end{array}$ & $\begin{array}{l}0.9 \\
(0.0001)\end{array}$ & - \\
\hline \multicolumn{7}{|l|}{$\begin{array}{l}\text { Domain² } \\
\text { PAQLQ }\end{array}$} \\
\hline $\begin{array}{l}\text { Activity } \\
\text { Limitation }\end{array}$ & & & & - & & \\
\hline Symptoms & & & & $\begin{array}{l}0.7 \\
(0.0001)\end{array}$ & - & \\
\hline Emotions & & & & $\begin{array}{l}0.6 \\
(0.0001)\end{array}$ & $\begin{array}{l}0.7 \\
(0.0001)\end{array}$ & - \\
\hline
\end{tabular}

p- value in brackets

1 - according to Bonferroni adjustment significance level $p<0.001$

2 - according to Bonferroni adjustment significance level $p<0.008$ 
Table 5. HRQOL scores and lung function - Spearmans' correlations

\begin{tabular}{|c|c|c|c|c|c|}
\hline & \multicolumn{5}{|c|}{$\begin{array}{c}\text { Lung function test } \\
\text { (10 minutes after exercises test) }\end{array}$} \\
\hline & FEV1 & $\begin{array}{r}\text { FEV1 } \\
\text { \%FVC }\end{array}$ & FVC & PEF & $\begin{array}{c}\text { MEF } \\
25-75 \%\end{array}$ \\
\hline \multicolumn{6}{|l|}{ QLCCDQ domain } \\
\hline \multicolumn{6}{|l|}{ Role functioning } \\
\hline Family roles & 0.5 & 0.3 & 0.1 & -0.3 & 0.8 \\
\hline Social roles & 0.1 & 0.01 & 0.07 & -0.1 & 0.8 \\
\hline $\begin{array}{l}\text { Occupational } \\
\text { roles }\end{array}$ & 0.2 & 0.3 & 0.1 & 0.2 & 0.2 \\
\hline $\begin{array}{l}\text { Roles activity } \\
\text { limitation }\end{array}$ & 0.2 & 0.3 & 0.1 & 0.2 & 0.6 \\
\hline Symptoms & 0.06 & 0.6 & -0.02 & 0.2 & 0.2 \\
\hline Emotions & 0.02 & 0.7 & -0.09 & 0.2 & 0.2 \\
\hline \multicolumn{6}{|l|}{ PAQLQ domain } \\
\hline Symptoms & 0.01 & 0.2 & 0.4 & 0.2 & 0.2 \\
\hline Activity limitation & 0.2 & 0.2 & 0.4 & 0.5 & 0.4 \\
\hline Emotions & -0.05 & 0.2 & 0.2 & 0.09 & 0.2 \\
\hline
\end{tabular}

